\title{
Total Cost of Ownership Optimization of Manufacturing Machines with Fast Energy Storage
}

\author{
Bert Lenaerts*, Ahmed Abdallh*, Davy Maes*, Branimir Mrak*, Timothy Galle ${ }^{\dagger}$, Wim De Waele ${ }^{\dagger}$ \\ * Flanders Make vzw, 3001 Leuven, Belgium \\ E-mail: \{firstname.lastname\}@ flandersmake.be \\ $\dagger^{\dagger}$ Ghent University, 9000 Ghent, Belgium \\ E-mail: \{firstname.lastname\}@ugent.be
}

\begin{abstract}
Optimization of total cost of ownership (TCO) is an important, and challenging design target for present day manufacturing machines. This paper is concerned specifically with production machines with fast reciprocating loads $(>1 \mathrm{~Hz})$, e.g. weaving looms and plate punching machines. Subsequent acceleration and deceleration give rise to a reciprocating energy flow that can be handled either mechanically or electrically. The chosen solution will affect the total cost of ownership. In addition to the cost of the energy storage device itself, there are the energy bill, the size and cost of the electric drive and power supply to consider. Moreover, there are certain constraints to be met: lifetime, DC-bus voltage limits and total power factor. This paper presents a methodology that takes all these aspects into account. It applies it to a bar linkage mechanism, which is representative for the targeted applications. In the mechanical domain, springs are considered for energy storage. The structural design of the spring is included in the analysis in order to account for lifetime and inertia added by the spring. In the electric domain, three different topologies are compared: a purely passive front end, where energy is stored directly on the DC-bus, a passive front end combined with a DC/DC converter and a separate storage capacitor, and an active front end.
\end{abstract}

\section{INTRODUCTION}

Optimization of the total cost of ownership (TCO) has gained a lot of attention during the last decade in scientific and industrial research [1], [2]. Many original equipment manufacturers (OEMs) realize that increasing the energy efficiency of their drive trains offers the greatest leverage in reducing the TCO [3]. For example, recovering the regenerative energy in vehicles by means of electrification has become a pivotal goal in automotive industry [4], [5], [6]. Also in crane and elevator applications, there is a shifting focus towards energy recuperation [7], [8].

This paper studies the effect of energy storage components on the TCO of manufacturing machines. Manufacturing machines often exhibit significant energy flows converted back and forth between the electrical and mechanical domain. What sets them apart from electric vehicles, cranes and elevators, is the rate at which energy is converted. A full motion cycle takes less than 1 second, maybe only $100 \mathrm{~ms}$, for a weaving or platepunching application. Compared to the 'slow' energy storage of elevators and cranes, these kind of applications exhibit a much higher power to energy ratio. Hence, different solutions may be expected from a TCO optimization.

\section{SCOPE AND APPROACH}

The targeted applications are machines with periodic motions at high frequencies $(>1 \mathrm{~Hz})$, that result in a high alternating to average power ratio $(>2)$. The machines comprise an electric drive that is supplied through a three-phase, $400 \mathrm{~V}$ grid.

\section{A. TCO Optimization}

The purpose of this work is to minimize the TCO of the machine's drive train, while complying with all system requirements. This comes down to solving the following optimization problem:

- Objective function: the TCO over a specified period of time, usually the amortization period of the machine.

- Design variables: the parameters of the different components, like motors, drives, springs, capacitors, inductors, converters, as well as the exact trajectory followed by the machine.

- Constraints: trajectory requirements, lifetime, DC-bus voltage limits and total power factor.

In order to deal with the rather large design space, the problem is split up into different stages in each of which a certain set of parameters is optimized. This may be done iteratively when dependencies exist between different sets, as will be detailed below.

To start with, the problem is split up into a mechanical and an electrical optimization exercise. While this is sensible, and certainly convenient, it remains an approximation in the sense that the power factor constraint is not directly included in the mechanical domain optimization.

\section{B. A Note on Power Factor}

Because it is difficult to assess the exact costs associated with a low power factor, it is included as a constraint function in the TCO optimization rather than a cost. While the displacement power factor is typically very good $(>0.95)$ for the solutions discussed below, the total power factor can be undesirably low, due to harmonic distortion and load modulation. The contribution of the latter is especially important for the applications targeted here.

It should be noted that additional charges for reactive power by distribution companies, relate to displacement power factor only. At least this is the present day situation in all countries 

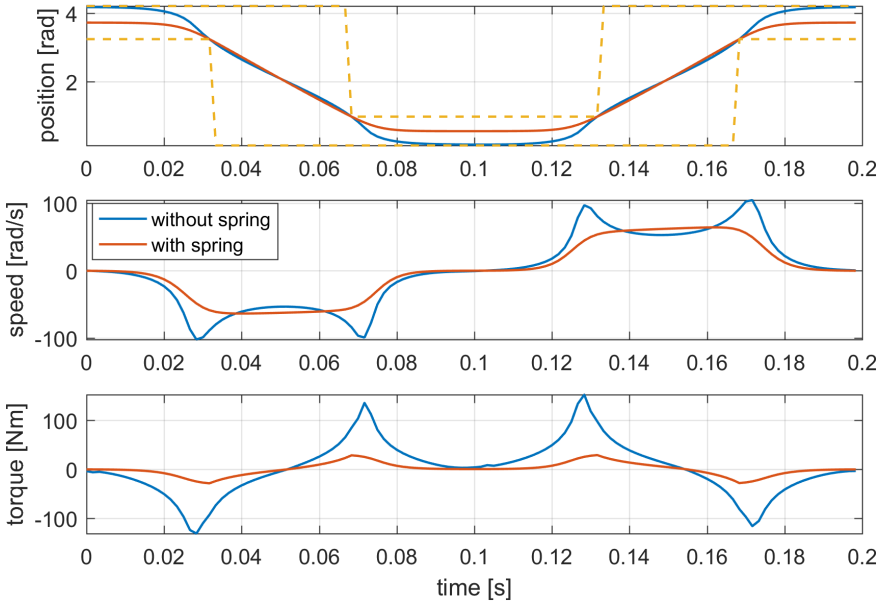

Fig. 1: Optimized crank trajectory for start-stop operation. The constraints are plotted in dashed line.

where the authors inquired with the distribution companies, i.e. Belgium, Egypt and Malaysia.

\section{Test Case: Bar Linkage Mechanism}

The bar linkage mechanism is representative for many manufacturing machines: a constant speed at the input (crank) translates into a reciprocating motion at the output (rocker or slider). Alternative transmissions may be encountered that serve the same purpose, for instance cam follower mechanisms.

The dimensions of the reference mechanism are such that a full crank rotation yields a rocker stroke of $+/-12^{\circ}$. Combined with a rocker inertia of $2 \mathrm{kgm}^{2}$, this yields a peak power of $10 \mathrm{~kW}$ at a constant crank speed of 10 revolutions per second.

Apart from constant speed operation, the mechanism is also required to operate in start-stop mode, as illustrated in figure 1. The exact trajectory is not important for the correct functioning of the machine, as long as the position vs. time constraints, also indicated, are met.

The speed range of the machine is 5 to $10 \mathrm{~Hz}$ for continuous operation or 2.5 to $5 \mathrm{~Hz}$ for start-stop operation. Furthermore, the machine should be able to operate in slow motion.

A reducer is installed between motor and crank. The reduction ratio is 4 and is not included as a design variable here. The motor itself - a high-dynamic, water cooled permanent magnet synchronous machine - is a dependent design variable. A scalable equivalent circuit and motor drive model are foreseen.

On the electrical side, a total power factor of at least 0.8 is required. The lifetime should be at least 50000 hours, both for mechanical and electrical components.

\section{Mechanical domain Optimization}

\section{A. Modeling and Optimization}

As already mentioned section III, there exists an energyoptimal trajectory for a given design and set of trajectory constraints. Figure 1 illustrates how the optimal trajectory may

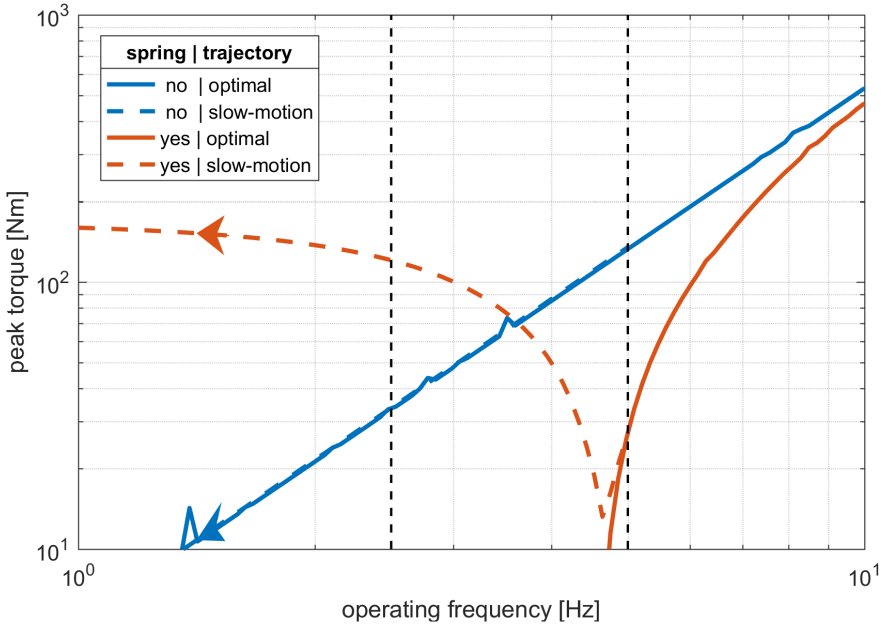

Fig. 2: Crank peak torque versus operating frequency.

differ for sprung and unsprung designs. Minimal RMS torque is pursued, which is approximately equivalent to minimal losses.

The kinematic relations of a four bar linkage are used for calculating torques [9]. A nonlinear least squares solver is employed for optimizing the trajectory [10]. Everything is implemented in MATLAB ${ }^{\circledR}$, making use of the OPTI toolbox for calling the solvers [11].

Figure 2 plots the peak torque delivered on the crank for different operating frequencies. If the frequency drops, so does the required peak torque. For the spring assisted case, the required torque quickly becomes negligible, since the natural motion of the inertia-spring system meets the constraints. The actuator's only task is to lock the mechanism in the dead points, where the spring torque exercised on the crank is zero. This is the case where the trajectory is optimal. If the trajectory remains fixed, like is required for slow motion operation, the actuator works against the spring. The slow motion requirement sets a limit on the spring stiffness: too stiff a spring leads to an expensive motor and drive.

The complete optimization approach is clarified in figure 3 . It consists of three nested loops. The top-level objective is a minimal total cost of ownership of the machine over 20000 hours of operation (note that the expected lifetime is 50000 hours). NOMAD is used as optimizer here [12]. Trajectory optimization is carried out using the NL2SOL solver [10]. The required motor, and its corresponding inertia, is found through iteration.

\section{B. Structural Spring Design}

As the required design lifetime of the components exceeds $10^{7}$ loading cycles, they are subjected to so-called very high cycle fatigue (VHCF). In this case fatigue behaviour is controlled by subsurface crack nucleation and initiation due to the presence of non-metallic inclusions and inhomogeneities in high-strength steels and alloys [13]. A safe life design based on infinite life is not possible anymore due to the absence of a fatigue endurance limit. Several models have been 


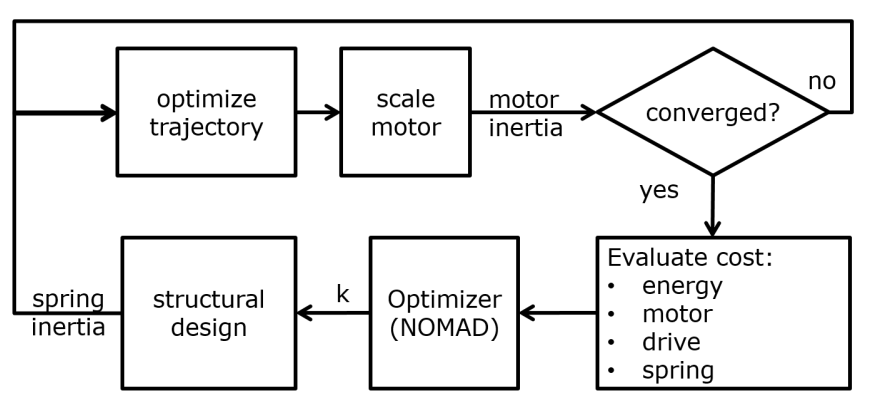

Fig. 3: Nested optimization approach for finding an optimal spring: the outer loop proposes a spring constant and evaluates the TCO. For each proposed spring constant, a structural design of the spring is made. A lower loop finds the minimal motor (and drive) for a given trajectory. The lowest loop optimizes the trajectory for each different motor proposed.

developed to predict the S-N curve and/or fatigue lifetime in the VHCF regime [14]. In this work, the model of Murakami [14] based on inclusion size and hardness of the metal has been implemented as a reference, as well as more recent approaches [15], [16] derived from the reference model and based on vast experimental evidence for a range of contemporary steels and alloys.

Given a required stiffness and deflection angle, a structural design is made for the rotative spring. Two spring types are investigated: the torsion bar and the spiral spring. Their optimal designs are given in table I for both options. They were found by means of an optimization routine around the well known spring equations, as can be found in [17] for instance. The required lifetime of 50000 hours translates into very low allowable stress levels and consequently large spring designs. For comparison, the designs for VHCF (50000 hours) and for conventional high cycle fatigue (500 hours) are included. Springs are assumed to be made of carbon steel valve spring wire EN10270-2 VDC.

The torsion bar design has only two geometrical parameters: diameter and length. The length/diameter ratio is directly proportional to the required deflection angle. Since there are practical limits on this ratio, the amount of deflection that can be achieved with a torsion bar is inherently limited $\left(+/-15^{\circ}\right.$ for an aspect ratio of 100). The strengths of the torsion bar are its low inertia and its symmetrical nature that allows for bidirectional loading.

The spiral spring has three additional degrees of freedom (number of turns, steel thickness and inner diameter) that can be exploited to obtain larger deflection angles and more practical dimensions. The downside is an increased moment of inertia. Another important limitation of the spiral spring is that it can be loaded in compression only. In the bar linkage application, two preloaded spiral springs have to be installed in anti-parallel, or some rectifying mechanism has to be foreseen.

\section{Total Cost of Ownership}

The cost models for motor, gearbox and drive (lifetime = 60000 hours) are based on supplier quotations for volumes $>$
TABLE I: Structural spring designs for different lifetime requirements

\begin{tabular}{llcccc}
\hline & & \multicolumn{2}{c}{ torsion bar } & \multicolumn{2}{c}{ spiral spring } \\
\hline lifetime & {$[\mathrm{h}]$} & 50000 & 500 & 50000 & 500 \\
stiffness & {$[\mathrm{kNm} / \mathrm{rad}]$} & 7.2 & 7.2 & 5.9 & 5.9 \\
deflection & {$\left[{ }^{\circ}\right]$} & 12 & 12 & 12 & 12 \\
length & {$[\mathrm{mm}]$} & 3521 & 544 & 115 & 508 \\
diameter & {$[\mathrm{mm}]$} & 43 & 27 & 360 & 96 \\
inertia & {$\left[\mathrm{kgm}^{2}\right]$} & 0.009 & 0.0002 & 0.78 & 0.0016 \\
\hline
\end{tabular}

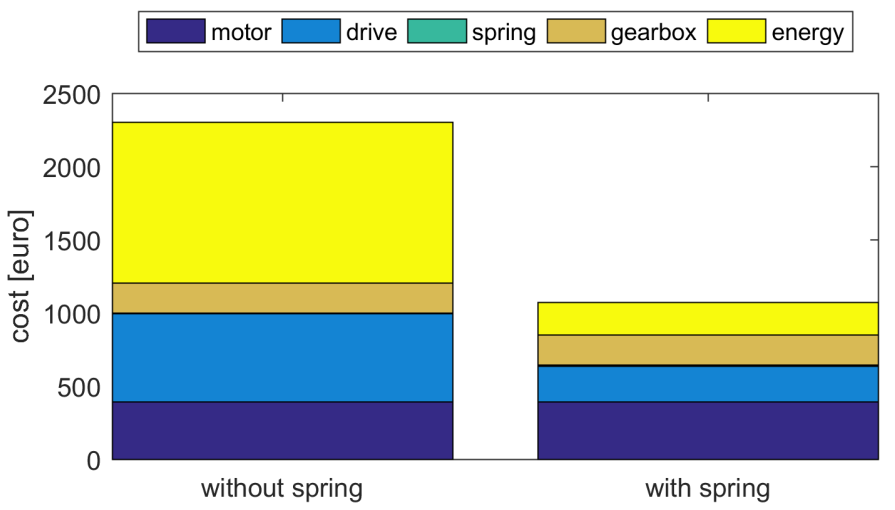

Fig. 4: Total cost of ownership for bar linkage test case when operated for 20000 hours in start-stop mode, at $5 \mathrm{~Hz}$.

1000. The cost of the motor is proportional to its peak torque, the cost of the drive proportional to its current rating. For the spring (torsion bar), an estimate is made that includes only material and tooling costs.

Since there is no useful mechanical power included in the model, all energy consumption is due to losses in the motor, drive and reducer. Motor losses are calculated from the trajectory and the (scaled) motor parameters. Drive losses are calculated from the motor currents and some generic drive parameters. The reducer losses are approximated by a fixed efficiency of 0.96 .

The total cost of ownership for the optimized solutions of the bar linkage test case is given in figure 4 . It is clear that significant cost savings may be realized with the use of a spring.

\section{Electrical Domain Optimization}

Three electrical front end topologies are considered for supplying power from the 3-phase grid to the DC-bus. The objective function is again total cost of ownership. The constraints are the DC-bus voltage limits $(500-750 \mathrm{~V})$, the total power factor (0.8) and the expected lifetime (50000 hours). The DC-bus voltage rating is $800 \mathrm{~V}$ and is realized with 400 V components (two in series for each parallel branch). Only capacitors are considered as lifetime critical components.

\section{A. Passive Front End}

This is the cheapest, most commonly encountered front end topology. As depicted in figure 5a, it consists of a line reactor, a six-pulse diode rectifier and a DC-bus capacitor. The two 


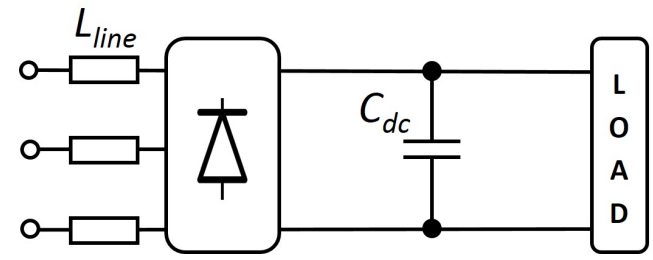

(a) Passive Front End

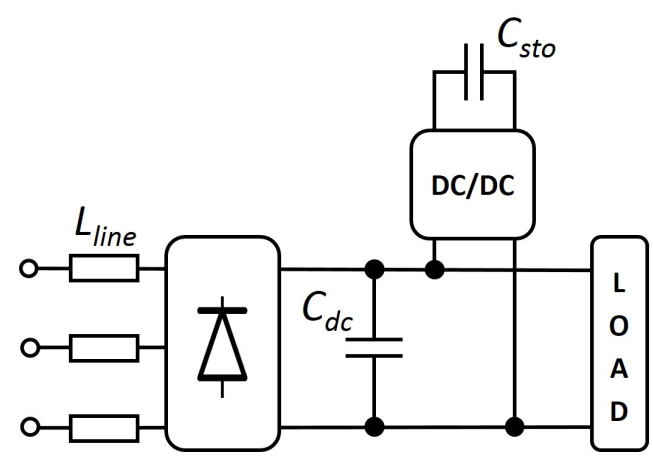

(b) Passive Front End + DC/DC converter

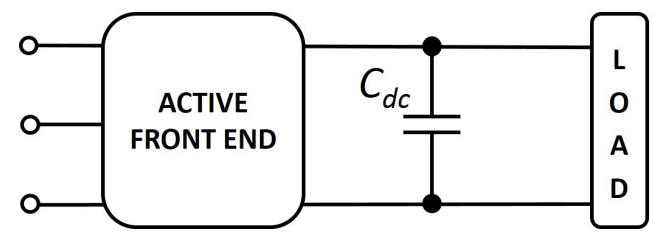

(c) Active Front End

Fig. 5: The three electrical topologies.

main design variables are the line reactor's inductance $L_{\text {line }}$ and the DC-bus capacitance $C_{d c}$.

A physical model is implemented in Simscape ${ }^{\mathrm{TM}}$. One of the inputs to this model is the electrical load profile that is obtained from the mechanical domain simulations discussed above. An optimizer is called to find the $L_{\text {line }}-C_{d c}$ combination that meets the constraints at minimal cost [12]. Figure 6 depicts the resulting DC-bus voltage and grid current in an optimized PFE for the bar linkage mechanism without spring.

The DC-bus provides the energy storage for regenerative braking and acceleration. It has to be sufficiently large for not violating the DC-bus voltage constraints. A higher capacitance also enhances the continuity of the power taken from the grid and hence the total power factor. Finally, a higher capacitance has more chance of meeting the lifetime constraints, since a similar RMS current is distributed over more cells.

The line reactor contributes to a better power factor by increasing the conduction angle of the diodes and reducing the harmonic content. On the other hand, a line reactor comes with a voltage drop, so too high an inductance will violate the constraint on minimum DC-bus voltage.
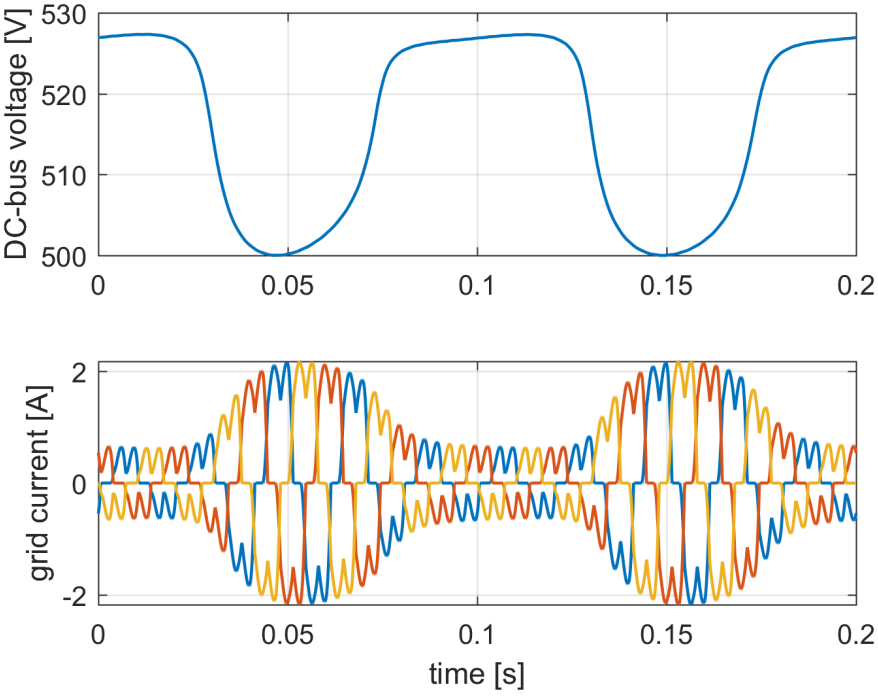

Fig. 6: DC-bus voltage and grid currents for the test case without spring, with cost-optimal passive front end $\left(C_{d c}=9.2\right.$ $\mathrm{mF}, L_{\text {line }}=50 \mathrm{mH}, \mathrm{PF}=0.8$ ).

\section{B. Passive Front End $+D C / D C$ converter}

The passive front end topology is extended with a DC/DC converter that sources/sinks power from the DC-bus to a separate storage capacitance, as depicted in figure $5 \mathrm{~b}$. This is a popular topology in electric vehicles - although no PFE is involved here - and 'slow' energy storage applications, like cranes and elevators [4], [5], [8], [18], [19].

Large voltage variations can be allowed on the storage capacitor to better utilize its energy storage potential. With a DC/DC converter that supplies the full peak power, the DC-bus voltage can be kept constant without the need for additional DC-bus capacitance. The cost is hence shifted from passive component cost (capacitor) to active component cost (DC/DC converter).

Again, a Simscape ${ }^{\mathrm{TM}}$ model is built that has the electrical load profile as input. The DC/DC converter matches the alternating part of the load profile up to its peak current rating. This topology has three design variables: the line inductance $L_{\text {line }}$, the storage capacitance $C_{\text {sto }}$ and the current rating of the DC/DC converter. Again, NOMAD is called to find the design that meets all constraints at minimal cost. $C_{d c}$ is not part of the optimization problem, although a minimal value is assumed for filtering the six-pulse rectifier output.[12].

\section{Active Front End}

If an active front end is used instead of a passive front end, as depicted in figure $5 \mathrm{c}$ the energy flow to the grid is controllable and bidirectional. This added controllability can be exploited in two different ways:

1) The DC-bus voltage is kept constant. In this case, the connected grid is used for energy storage. Regenerative braking and acceleration power is delivered through the active front end. Much like the PFE + DC/DC topology, this strategy is commonly encountered in 'slow' energy 
storage applications [7], [20], [21]. The average power drawn from the grid is low, while the apparent power is high, resulting in an overall bad power factor.

2) The power from the grid is kept constant and equal to the average required power. Braking and acceleration power is delivered through the DC-bus capacitor. The DC-bus voltage may fluctuate heavily, but the power from the grid remains constant, as opposed to a passive front end topology. Moreover, the grid currents drawn by the active front end are sinusoids, and ideally a power factor of 1 is obtained. The active front end in this case is sized for average power only, and not for peak power.

It is found that option 2 is the natural choice for fast energy storage applications. The available cost models (see V-E) show that the cost of increasing the current rating of the active front end exceeds that of installing additional DC-bus capacitance. This is concluded not only for the test case as described in III, but for a range of representative load profiles (frequency $>1 \mathrm{~Hz}$, alternating power / average power $>2$ ).

In some applications, starting currents may be several factors higher than what is needed in normal operation. In that case, a low-cost diode rectifier, without line reactor, might be installed in parallel with the AFE to accommodate these currents, as such avoiding an increased AFE cost.

\section{Capacitor Lifetime}

Only capacitor lifetime is considered critical and is explicitly modeled. Well established lifetime models are available for aluminum electrolytic capacitors, which is the de facto standard technology for DC-buses [22]. These lifetime models take the simulated current profiles as input, as well as the ambient temperature, which is set to $45^{\circ} \mathrm{C}$. Predicted lifetimes were validated using the lifetime prediction tools of the capacitor manufacturers themselves [23], [24].

\section{E. Cost Comparison}

The TCO of the three topologies for the bar linkage test case is given in figure 7 . The energy cost is not included, although some minor differences exist due to additional losses in the DC/DC converter and active front end. As already explained in II-B, only the displacement power factor is relevant for the electricity cost. Since it is well above 0.95 in all cases, it does not raise any additional charges.

The DC/DC converter cost model is based on [25], the capacitor cost model on [26]. The front end cost models, including line reactors/filters, are based on supplier quotations for volumes $>1000$.

This kind of analysis was carried out not only for the test case described in III, but for a range of representative load profiles, all periodic with a fundamental frequency $>1 \mathrm{~Hz}$. The topology with DC/DC converter was consistently found more expensive than the passive front end solution. This is in line with the observation that the converter cost dominates over storage cost for a high power to energy ratio (fast energy storage).

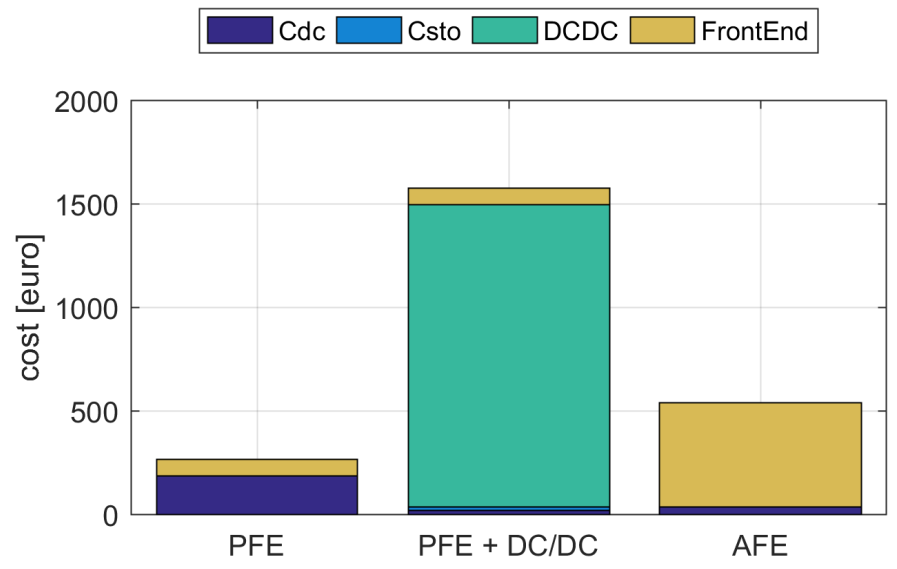

(a) without spring

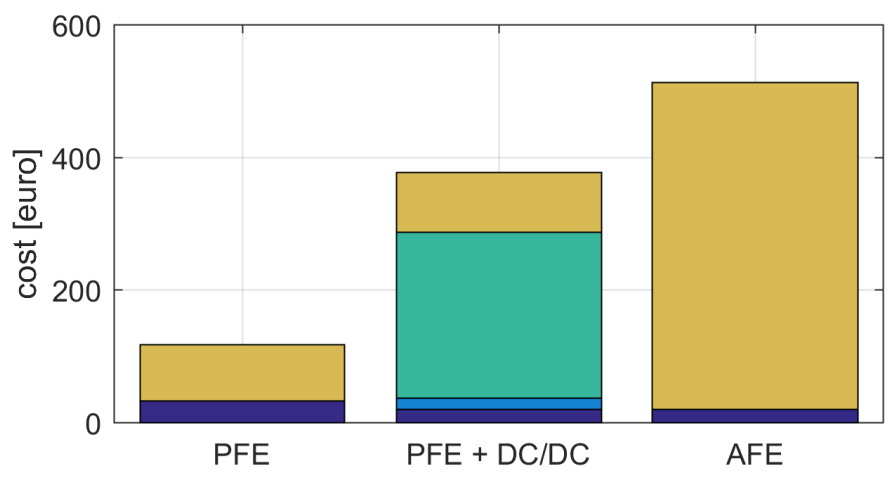

(b) with spring

Fig. 7: Cost comparison of three electrical topologies for the bar linkage test case, with and without spring assistance. The front end cost includes line reactors (PFE) and filter sections (AFE). Each topology has been optimized for cost, while meeting voltage, power factor and lifetime constraints. The AFE solution achieves a power factor of 1 , the others the required minimum of 0.8 .

The active front end solution differs from the DC/DC solution in this respect. It delivers the average power only, while the oscillating power flow is handled by the DC-bus. Where high total power factors $(>0.9)$ are required, an active front end becomes more cost-effective than a passive solution. As an additional benefit, an active front is less sensitive to grid unbalance and voltage drops. Furthermore, the boosted DC-bus voltage may extend the electric drive's feasible speed-torque range.

\section{CONCLuSions}

An approach for optimizing the total cost of ownership of manufacturing machines with energy storage components has been presented and applied to a representative test case: a bar linkage mechanism operated in start-stop mode. Both mechanical (springs) and electrical (capacitors) storage components are considered.

It is shown that a parallel spring on the rocker of the four bar linkage leads to a substantial reduction in total cost 
of ownership: both component and energy cost are reduced. The lifetime requirement and added inertia of the spring are included in the analysis through a structural spring design. The torsion bar design seems most suited, although its dimensions may be unpractical. To achieve gigacycle lifetime, the deflection angle of the torsion bar is inherently limited to $+/-15^{\circ}$ for a length to diameter ratio of 100 . The spiral spring design does not exhibit this limitation, but has other drawbacks, like a higher inertia and unidirectional operation.

It is found that a DC/DC converter with a separate storage capacitor, as encountered in vehicle and crane applications, is not cost-effective for fast energy storage. Given the high frequencies involved, it is cheaper to store energy directly on an upscaled DC-bus. This finding has been consistently reproduced for a wide range of load profiles $(>1 \mathrm{~Hz})$.

An active front end may be a cost-effective solution where high power factors $(>0.9)$ are required. The proposed approach is to have the active front end provide the average power only, and size it accordingly. The ( $>2$ times) higher, alternating power is handled by the DC-bus. Advantages inherent to the active front, a higher DC-bus voltage and insensitivity to grid unbalance and voltage drops, are a bonus.

\section{ACKNOWLEDGMENT}

This work is performed as part of the PROFENSTO_icon project, funded by the Institute for the Promotion of Innovation through Science and Technology in Flanders (VLAIO), Belgium.

\section{REFERENCES}

[1] S. Thiede, T. Spiering, and S. Kohlitz, "Dynamic Total Cost of Ownership (TCO) Calculation of Injection Moulding Machines," Leveraging Technology for a Sustainable World, pp. 275-280, 2012. [Online]. Available: http://link.springer.com/chapter/10.1007/978-3-64229069-5_47

[2] I. Roda and M. Garetti, "TCO Evaluation in Physical Asset Management: Benefits and Limitations for Industrial Adoption," in IFIP Advances in Information and Communication Technology, vol. 440, no. PART 3, 2014, pp. 216-223.

[3] P. Wikstroem, J. Tolvananen, A. Savolainen, and P. Barbosa, "Saving energy through drive efficiency," 2007.

[4] A. F. Burke, "Batteries and Ultracapacitors for Electric, Hybrid, and Fuel Cell Vehicles," Proceedings of the IEEE, vol. 95, no. 4, pp. 806-820, apr 2007. [Online]. Available: http://ieeexplore.ieee.org/document/4168012/

[5] J. Moreno, M. Ortuzar, and J. Dixon, "Energy-management system for a hybrid electric vehicle, using ultracapacitors and neural networks," IEEE Transactions on Industrial Electronics, vol. 53, no. 2, pp. 614-623, apr 2006. [Online]. Available: http://ieexplore.ieee.org/document/1614145/

[6] J. Hagman, S. Ritzén, J. J. Stier, and Y. Susilo, "Total cost of ownership and its potential implications for battery electric vehicle diffusion," Research in Transportation Business \& Management, vol. 18, pp. 11-17, 2016. [Online]. Available: http://linkinghub.elsevier.com/retrieve/pii/S2210539516000043

[7] N. Mitrovic, M. Petronijevic, V. Kostic, and B. Jeftenic, "Electrical Drives for Crane Application," in Mechanical Engineering. InTech, apr 2008. [Online]. Available: http://www.intechopen.com/books/mechanicalengineering/electrical-drives-for-crane-application

[8] P. J. Grbovic, "Ultra-capacitor based regenerative energy storage and power factor correction device for controlled electric drives Dispositif correcteur de facteur de puissance à base de supercondensateur pour variateur de vitesse," jul 2011. [Online]. Available: https://tel.archives-ouvertes.fr/tel-00585405/
[9] J. M. Mccarthy, "Four-Bar Linkage Analysis: The 4R Quadrilateral," UC Irivine, The Henry Samueli School of Engineering, Tech. Rep., 2009. [Online]. Available: https://synthetica.eng.uci.edu/mechanicaldesign101/McCarthyNotes2.pdf

[10] J. E. Dennis, D. M. Gay, and R. E. Welsch, "Algorithm 573: NL2SOL-An Adaptive Nonlinear Least-Squares Algorithm [E4]," ACM Transactions on Mathematical Software, vol. 7, no. 3, pp. 369-383, sep 1981. [Online]. Available: http://portal.acm.org/citation.cfm?doid=355958.355966

[11] J. Currie and D. I. Wilson, "OPTI: Lowering the Barrier Between Open Source Optimizers and the Industrial MATLAB User," in Foundations of Computer-Aided Process Operations, N. Sahinidis and J. Pinto, Eds., Savannah, Georgia, USA, 2012.

[12] M. A. Abramson, C. Audet, G. Couture, J. E. Dennis, Jr., S. Le Digabel, and C. Tribes, "The $\{$ NOMAD $\}$ project," Software available at \url\{https://www.gerad.ca/nomad/ $\}$. [Online]. Available: https://www.gerad.ca/nomad/

[13] Q. Wang, M. K. Khan, and C. Bathias, "Current understanding of ultra-high cycle fatigue," Theoretical and Applied Mechanics Letters, vol. 2, no. 3, jan 2012. [Online]. Available: https://www.sciencedirect.com/science/article/pii/S2095034915301434

[14] Y. Murakami and M. Endo, "Effects of defects, inclusions and inhomogeneities on fatigue strength," International Journal of Fatigue, vol. 16, no. 3, pp. 163-182, apr 1994. [Online]. Available: https://www-sciencedirectcom.kuleuven.ezproxy.kuleuven.be/science/article/pii/0142112394900019

[15] C. S. Bandara, S. C. Siriwardane, U. I. Dissanayake, and R. Dissanayake, "Fatigue Strength Prediction Formulae for Steels and Alloys in the Gigacycle Regime," International Journal of Materials, Mechanics and Manufacturing, vol. 1, no. 3, pp. 256-260, 2013.

[16] Y. Liu, Y. Li, S. Li, Z. Yang, S. Chen, W. Hui, and Y. Weng, "Prediction of the SN curves of high-strength steels in the very high cycle fatigue regime," International Journal of Fatigue, vol. 32, no. 8, pp. 1351-1357, aug 2010. [Online]. Available: https://www-sciencedirectcom.kuleuven.ezproxy.kuleuven.be/science/article/pii/S0142112310000459

[17] MITCalc, "Springs Calculation." [Online]. Available: http://www.mitcalc.com/doc/springs/help/en/springs.htm

[18] M. P. Shreelakshmi and V. Agarwal, "An Energy Efficient and Environment Friendly Elevator System Using Ultracapacitor and Fuel Cell with Power Factor Correction," in 2013 IEEE ECCE Asia Downunder. IEEE, jun 2013, pp. 721-727. [Online]. Available: http://ieeexplore.ieee.org/document/6579181/

[19] A. Kumar Chakrawarti, R. Singh, and M. Scholar, "Review of Ultracapacitor application in energy saving Elevator System," International Journal Online of Science, vol. 3, no. 9, 2017. [Online]. Available: http://ijoscience.com

[20] V. Beldjajev, T. Lehtla, and H. Mõlder, "Influence of regenerative braking to power characteristics of a gantry crane," in PQ2010: 7th International Conference - 2010 Electric Power Quality and Supply Reliability, Conference Proceedings, 2010, pp. 73-77.

[21] K. R. Rasin and G. Arunkumar, "Regeneration in Variable Frequency Drives and Energy saving Methods," International Research Journal of Engineering and Technology(IRJET), vol. 4, no. 3, pp. 1246-1249, 2017. [Online]. Available: https://irjet.net/archives/V4/i3/IRJET-V4I3295.pdf

[22] M. Frivaldsky, M. Pridala, and P. Drgona, "Implementation of mathematical model of thermal behavior of electronic components for lifetime estimation based on multi-level simulation," Archives of Electrical Engineering, vol. 66, no. 2, pp. 339-350, 2017. [Online]. Available: http://www.degruyter.com/view/j/aee.2017.66.issue2/aee-2017-0025/aee-2017-0025.xml

[23] CDE, "Cornell Dubilier Thermal / Life Calculators." [Online]. Available: http://www.cde.com/STapplet/CDE_Applet_Intro.html

[24] KEMET, "Electrolytic Life Calculator." [Online]. Available: http://elc.kemet.com/

[25] A. Kulkarni, W. Chen, and A. Bazzi, "Implementation of Rapid Prototyping Tools for Power Loss and Cost Minimization of DC-DC Converters," Energies, vol. 9, no. 7, p. 509, jul 2016. [Online]. Available: http://www.mdpi.com/1996-1073/9/7/509

[26] R. Burkart and J. W. Kolar, "Component cost models for multi-objective optimizations of switched-mode power converters," in 2013 IEEE Energy Conversion Congress and Exposition. IEEE, sep 2013, pp. 2139-2146. [Online]. Available: http://ieeexplore.iee.org/document/6646971/ 